\title{
L'évolution de l'encépagement des indications géographiques françaises : outils de gestion du vignoble et contraintes subies
}

\author{
Jacques Gautier ${ }^{1, \text { a }}$ et Eric Rosaz ${ }^{2, b}$ \\ ${ }^{1}$ INAO, Inspecteur national, 1 Quai Wilson, 33130 Begles, France \\ 2 INAO, Responsable Pôle, « Vins, cidres et boissons spiritueuses », 12 rue Rol -Tanguy, TSA 30003, 93100 Montreuil, \\ France
}

L'encépagement des vignobles français et plus particulièrement des indications géographiques (IG) françaises a évolué à travers le temps. Pour répondre et s'adapter aux différents enjeux et contraintes de leur environnement, les producteurs français ont sensiblement modifié leurs pratiques culturales, ouvert de nouvelles voies, fait évoluer leur encépagement dans le cadre réputé contraint que sont les indications géographiques.

Le choix de l'encépagement est une composante essentielle dans l'établissement d'un vignoble, et la liste des cépages ouvrant droit au bénéfice d'une IG est décrite précisément dans le cahier des charges de celle-ci.

Nous examinerons successivement les questions suivantes :

- Quels sont les principaux facteurs ayant agit par le passé sur l'évlution de l'encépagement des IG ?

- Quelles sont les perspectives à moyen et à long terme, en réponse aux problématiques actuelles comme notamment les évolutions climatiques, le souhait de réduire les intrants ou encore les attentes sociétales sur les teneurs en alcool des vins bénéficiant d'une indication géographique?

$$
\text { * }
$$

En charge depuis sa création en 1935 de la gestion des indications géographiques françaises, l'Institut national de l'origine et de la qualité (INAO) a pour mission de définir les conditions de production des indications géographiques lors de leur reconnaissance, et notamment leur encépagement. L'INAO est également régulièrement sollicité par les producteurs pour modifier ces conditions de production.

Les instances de gouvernance professionnelle de l'INAO, et plus particulièrement celles en charge du secteur des appellations d'origine contrôlées, ont établi des règles pour le traitement de ces demandes, demandes qui devraient dans un avenir proche être amenées à se développer vu les travaux de recherche menés actuellement sur l'obtention de nouveaux cépages.

Le CTPS (Comité technique permanent de la sélection), instance de pilotage du catalogue officiel

a e-mail : j.gautier@inao.gouv.fr

b e-mail : e.rosaz@inao.gouv.fr français des variétés, instruit prioritairement l'inscription de ces nouveaux cépages, dans le respect d'une méthode validée par les scientifiques.

\section{Situation des vins français sous indication géographique, AOP et IGP}

Pour leur très grande majorité (plus de $90 \%$ des volumes), les vins français bénéficient d'une indication géographique, dans une des deux catégories suivantes, appellations d'origine contrôlée (AOC/AOP) et indications géographiques protégées (IGP) :

A - Créées en 1935, les AOC reposent sur l'existence d'un lien fort entre le produit et son terroir. Le respect des différentes conditions de production définies précisément dans le cahier des charges de chaque AOC permet l'acquisition de caractéristiques bien particulières liées au terroir.

Le 26 juin 1948 le premier congrès national de l'origine rappelait les principes des appellations, dans la déclaration suivante :

«I - Un produit est d'origine lorsqu'il a une originalité propre, une personnalité consacrée par des usages et une renommée constatée.

Les qualités substantielles que doit présenter un produit d'origine résultent :

$1^{\circ}$ de facteurs naturels dont le rôle est prépondérant ; le climat, la nature du sol, l'exposition, la flore spontanée, les variétés végétales cultivées et races animales élevées. Ces facteurs se situent dans les limites d'une aire de production qui constitue la circonscription d'origine.

$2^{\circ}$ de facteurs dus à l'action continue du producteur qui contribue à l'affirmation et au développement de l'originalité du produit : méthodes de cultures, procédés de fabrication, de transformation et de conservation... »

Cette notion de qualité substantielle est primordiale, et avait fait l'objet de nombreux débats durant la période de constitution du système français d'appellation d'origine contrôlée au cours de la première moitié du $20^{\text {ème }}$ siècle. Deux thèses sur la notion d'origine avaient été largement débattues : celle basée sur l'origine et les qualités substantielles, et celle basée uniquement sur l'origine proprement dite.

Lors des discussions ayant précédé l'adoption de la loi du 6 mai 1919 donnant compétence au pouvoir judiciaire pour reconnaitre les appellations d'origine, un certain

This is an Open Access article distributed under the terms of the Creative Commons Attribution License 4.0, which permits unrestricted use, distribution, and reproduction in any medium, provided the original work is properly cited. 
nombre de décisions législatives et judiciaires ont entrainé la prééminence de la thèse basée sur la seule origine.

Il faudra attendre la création en 1935 du système des appellations d'origine contrôlées pour que la notion d'origine liée à une qualité substantielle soit définitivement retenue. En écartant clairement la thèse basée sur la seule indication de provenance, et en retenant le double fondement sur l'origine et les qualités substantielles, c'est véritablement une décision fondatrice qui a été retenue.

Elle aura permis l'émergence progressive du concept de «typicité »; elle constitue encore aujourd'hui le socle des indications géographiques dans leur conception désormais internationale et élargie à l'ensemble des filières agroalimentaires.

Avec leur expérience, les producteurs ont mis en avant des cépages présentant une adéquation particulière avec le milieu géographique de leur appellation et les usages de production mis en œuvre localement.

Certaines appellations sont dites monocépages, comme par exemple l'AOC Chablis avec le chardonnay B, les AOC du Muscadet avec le melon B, l'AOC Cour-Cheverny avec le romorantin B, l'AOC Picpoul de Pinet avec le piquepoul $\mathrm{B}$ ou encore certaines $\mathrm{AOC}$ privilégiant le cépage muscat à petits grains $\mathrm{B}$.

D'une manière générale le nombre de cépages ouvrant droit au bénéfice des AOC est restreint, même si les AOC méridionales peuvent présenter une liste plus importante.

Dans les cahiers des charges des AOC, l'encépagement peut être défini en trois catégories : les cépages principaux, les cépages complémentaires et les cépages accessoires. Des règles de proportion peuvent alors être fixées, les cépages principaux devant respecter un minimum des superficies revendiquant l'appellation, fréquemment supérieur à 50 ou $60 \%$ voire $80 \%$, les cépages complémentaires étant obligatoires mais dans une proportion généralement limitée à $40 \%$, alors que les cépages accessoires seront limités à une faible proportion de l'encépagement, généralement $10 \%$. Dans certains rares cas ce pourcentage sera même inférieur à $10 \%$, comme par exemple dans le cas de l'AOC Vouvray où le cépage orbois B est limité à $5 \%$ de l'encépagement de l'exploitation, le solde étant constitué par le cépage chenin B.

B - Les indications géographiques protégées (IGP), de conception beaucoup plus récente et s'appuyant principalement sur l'ancienne catégorie des « Vins de Pays 》 issue des vins de table, présentent un lien à l'origine moins établi que les AOC et se rapprochent donc de la conception basée sur la notion de provenance.

L'espace de liberté relatif aux conditions de production, et notamment l'encépagement, va en conséquence être beaucoup plus large. Le nombre de cépages offrant le droit au bénéfice de l'IGP est beaucoup plus important qu'en AOC.

\section{La définition retenue pour le 《cépage »}

La notion de cépage couramment utilisée dans le vignoble ne correspond pas à la notion de variété retenue par les botanistes (les cépages ne se reproduisant pas identiques à eux-mêmes par le semis). Il est donc apparu nécessaire de bien préciser la définition de la notion de cépage, afin de réduire les différences d'interprétation.

S'appuyant notamment sur les travaux de Jean-Michel BOURSIQUOT et Patrice THIS (ENSA Montpellier, INRA), le comité national en charge des AOC a statué sur cette question, pour adopter en 2009 la définition suivante du cépage :

« Le cépage, unité taxinomique propre à Vitis vinifera $L$, est le produit d'un semis ou d'un individu unique au départ, multiplié par voie végétative. Au cours des multiplications, des variations peuvent se produire et être fixées. Un cépage est donc composé d'un ensemble de clones suffisamment apparentés les uns aux autres pour être confondus sous un même nom et dont le nombre est variable (un ou plusieurs) et/ ou indéterminé. Lorsque la variation touche un caractère évident et remarquable ou ayant des conséquences technologiques importantes, le clone concerné est alors considéré comme une forme différenciée du cépage initial. »

Afin de mieux visualiser les orientations retenues, il est possible de se référer au schéma suivant reprenant la classification du règne végétal :

1 - Espèce (Vitis vinifera)

2 - Sous-espèce (ex : sativa)

3 - Unités - Divisions (ex : occidentalis)

4 - Familles - Groupes (ex : noiriens, carmenets, cotoïdes, traminers, ....)

5 - Cépages - Tribus (ex : pinot, merlot, grenache, ...)

6 - Cultivars - Formes (ex : pinot noir N, merlot noir N, ...)

7 - clones $(\mathrm{ex}: 115, \ldots)$.

La définition retenue pour le cépage correspond donc au niveau 6, celui du cultivar, niveau où la différenciation des génotypes repose sur la multiplication végétative (alors que pour les niveaux 1 à 5 la différenciation repose sur la voie sexuée).

Chaque cépage est constitué d'un certain nombre de clones, dont le nombre peut varier de façon importante.

\section{Evolution de l'encépagement en France : repères}

En 2013, sur près de 815000 hectares de vignes plantées en France, on recensait près de 170 cépages noirs et 145 cépages blancs, ce qui pourrait être interprété comme une grande diversité.

Toutefois, un examen plus précis montre que $74 \%$ du vignoble français est planté avec seulement 10 cépages, blancs et noirs confondus : merlot $\mathrm{N}$, grenache $\mathrm{N}$, ugni blanc B, syrah $\mathrm{N}$, cabernet sauvignon $\mathrm{N}$, chardonnay $\mathrm{B}$, carignan $\mathrm{N}$, cabernet franc $\mathrm{N}$, pinot noir $\mathrm{N}$ et sauvignon $\mathrm{B}$ sont les cépages les plus plantés du vignoble français.

Le merlot $\mathrm{N}$ est le cépage le plus planté, sur près de 115000 hectares. S'il se rencontre dans la presque quasitotalité des vignobles, on le retrouve plus particulièrement en Gironde et en Aquitaine.

L'ugni blanc B est le cépage blanc le plus planté en France, sur environ 83000 ha, avec une très forte implantation dans le vignoble charentais destiné à la production de l'AOC Cognac. 
L'évolution de l'encépagement ne peut se constater que sur des pas de temps importants. Malheureusement, les données disponibles en France sur des séries longues sont incomplètes et ne nous permettent pas d'être réellement pertinents dans notre approche.

Si en 15 ans, le poids des 20 premiers cépages blancs et noirs n'a pas véritablement varié, le nombre des différents cépages plantés est à noter : depuis 2000 , près d'une vingtaine de nouveaux cépages ont fait leur apparition.

Si à l'échelle nationale on ne constate que peu de variations de l'encépagement, il en va différemment dans un certain nombre de régions viticoles françaises où l'évolution et le contraste sont beaucoup plus marqués.

\section{Les demandes de modification de l'encépagement des indications géographiques}

L'encépagement du vignoble français a régulièrement connu de profondes modifications depuis la crise phylloxérique.

Ainsi on peut citer, à titre d'exemple, le cépage melon B qui a remplacé le chenin $\mathrm{B}$ en pays nantais, l'ugni blanc B qui a supplanté la folle blanche B dans la production de l'AOC Cognac, cépage qui avait lui -même supplanté antérieurement le colombard B, ou encore le « muscatel » exploité dans la région de Cassis pour la production de vins de liqueur qui a été supplanté par les cépages ugni blanc B, clairette B et marsanne B au profit d'une production de vins secs.

Par ailleurs des équilibres dans les proportions des différents cépages ont été largement modifiés, comme par exemple le large développement du merlot en Gironde avec un doublement des surfaces depuis 1979, alors qu'historiquement certaines zones du vignoble girondins étaient majoritairement plantées en cot $\mathrm{N}$ (dénommé localement malbec).

L'évolution de l'encépagement du vignoble bourguignon (Saône et Loire et Côte d'Or) est à ce titre également intéressante. Composé aujourd'hui principalement de pinot noir $\mathrm{N}$ et gamay $\mathrm{N}$ (cépages noirs) et de chardonnay $\mathrm{B}$ et dans une moindre mesure d'aligoté B (cépages blancs), le vignoble bourguignon a vu son encépagement profondément modifié depuis 1968 et cela sur deux aspects :

- la disparition de cépages considérés comme peu qualitatifs, représentant alors près de $30 \%$ du vignoble : gamay de Bouze $\mathrm{N}$, gamay de Chaudenay $\mathrm{N}$ (dits teinturiers), oberlin $\mathrm{N}$, baco $\mathrm{N}$ et plantet $\mathrm{N}$,

- une augmentation constante et importante de l'implantation du chardonnay B (près de 11000 ha aujourd'hui contre 5500 ha en 1968).

\subsection{Les premières orientations de l'INAO pour les AOC viticoles :}

Joseph CAPUS, le fondateur des AOC françaises, avait rappelé en 1947 dans l'introduction de l'ouvrage « Le Vignoble Girondin » (Lafforgue, Edition Louis LARMAT) que :
«Ici encore il n'est pas question d'usages. C'est la qualité et la notoriété seules des appellations qui doivent déterminer le Comité national (de l'INAO) dans son choix. Il est libre d'imposer les conditions qu'il jugera à propos des appellations qu'il contrôle, comme il est maitre de décider en quoi consiste la notoriété dont parle la loi. ...

Rien n'empêche le Comité national de dépasser les usages et, pour surélever la qualité, de se montrer aussi exigeant qu'il le faudrait pour accorder l'autorisation d'user d'une AOC. ....

Le Comité national est libre, dans les conditions de production qu'il impose, de choisir celles qu'il jugera opportun en vue de maintenir la qualité. Aucun usage ancien ne s'impose à lui. Ses décisions peuvent s'adapter à toutes les données de la science, à tous les progrès, qui sont de nature à améliorer la qualité des vins....».

Ces propos méritent d'être rappelés, d'une part parce qu'ils ont très largement été au centre de la pratique de nombreux acteurs des appellations d'origine depuis cette époque.

Mais ils démontrent aussi très clairement la volonté des fondateurs du système d'appellation d'origine contrôlée de prendre en compte le bénéfice des différentes évolutions techniques ; bien entendu sous réserve explicite du maintien de l'originalité et de la qualité des produits, c'està-dire de leur typicité.

L'objectif de l'INAO était de prendre des décisions favorisant l'amélioration de l'originalité et de la qualité des produits, et rien ne devait s'y opposer.

Une importante proportion du vignoble français était alors plantée avec des cépages très productifs, non qualitatifs, et l'objectif de la mise en place du système d'AOC était de s'assurer que la qualité des vins d'AOC ne serait pas affaiblie par une éventuelle incorporation, même en faible proportion, de ces cépages productifs.

Une partie du vignoble était également plantée avec des hybrides producteurs directs (HPD), dont les plantations étaient favorisées par leur résistance aux maladies. Mais les vins issus de ces cépages présentaient des caractéristiques dominées par des goûts foxés, incompatibles avec l'originalité et la qualité des vins d'AOC.

Dans l'objectif d'éradication de ces cépages HPD, l'INAO a largement utilisé la découverte de l'Institut d'œnologie de Bordeaux, qui avait mis en évidence que la présence de malvosides dans les vins était exclusivement liée aux HPD.

L'analyse systématique de l'éventuelle présence de malvosides dans les vins revendiquant une $\mathrm{AOC}$, va permettre à l'INAO d'éliminer dans les années 1970 1980 tous les HPD des exploitations viticoles productrices d'AOC.

L'utilisation de cette découverte a ainsi permis de compléter efficacement le contrôle des HPD réalisés à la vigne depuis le début des années 1960 par les agents de l'INAO. 


\subsection{Les demandes actuelles portant sur l'évolution de l'encépagement :}

Les objectifs des demandes de modification d'encépagement reçues récemment sont assez diversifiées, variant selon les situations de chaque appellation : corriger certaines caractéristiques des cépages actuels (manque de couleur, équilibres acidité/alcool des vins, ... ), modifier la productivité des cépages, réduire les risques liés à certains cépages (risque de coulure très marqué, sensibilité à la pourriture ou à certaines maladies), rechercher des cépages présentant un cycle végétatif permettant de décaler les dates de vendanges, ...

Ces demandes d'évolution de l'encépagement peuvent être classées en trois grandes catégories :

a) Les demandes visant à l'introduction de cépages de forte notoriété, déjà existants dans un certain nombre d'AOC, étrangers à la région viticole de l'AOC concernée par la demande.

Ces cépages de forte notoriété ont quelquefois été qualifiés «d'améliorateurs », ou tout au moins étaient supposés apporter un gain qualitatif.

L'étude approfondie de ces demandes montrent qu'elles ont pu s'inspirer de phénomènes de mode, comme cela a été observé avec les demandes d'introduction de ces cépages dans des régions où ils n'étaient pas implantés classiquement.

L'introduction de ces cépages visait en général à favoriser une amélioration sur quelques paramètres mesurables réputés 《qualitatifs ». Malheureusement cette introduction a conduit à une banalisation des types des vins produits, au détriment de leur originalité.

L'engouement observé pour certains cépages « nobles », largement prôné par quelques prescripteurs, a conduit à favoriser une uniformisation des caractéristiques des produits, uniformisation largement accentuée par le nombre restreint de ces cépages, parmi lesquels : chardonnay $\mathrm{B}$, sauvignon $B$, viognier $B$, marsanne $B$, roussanne $B$, vermentino $\mathrm{B}$ pour les vins blanc, merlot $\mathrm{N}$, syrah $\mathrm{N}$, cabernetsauvignon $\mathrm{N}$ ou cabernet franc $\mathrm{N}$ pour les vins rouges.

En outre les conséquences de cette uniformisation seront accentuées par la généralisation de certaines pratiques œnologiques ou la production de rendements inadaptés à l'expression du terroir.

b) Les demandes de cépages issus de croisements intraspécifiques entre différents cépages de l'espèce Vitis vinifera.

Des demandes ont porté sur l'introduction de nouveaux cépages improprement dénommés 《métis », puisque résultant de croisements entre cépages appartenant à l'espèce Vitis vinifera.

Les cépages concernés par ces demandes sont notamment le folignan B (folle blanche B x ugni blanc B) pour les AOC de la région de Cognac, le marselan $\mathrm{N}$ (cabernet sauvignon $\mathrm{N} x$ grenache $\mathrm{N}$ ) et le caladoc $\mathrm{N}(\cot \mathrm{N} x$ grenache $\mathrm{N})$, le couston $\mathrm{N}$ (grenache $\mathrm{N} x$ aubun $\mathrm{N}$ ) pour des AOC de la région sud de la France, ou encore le gamaret N (gamay $\mathrm{N} x$ reichensteiner $\mathrm{B}$ ) pour les $\mathrm{AOC}$ de la région beaujolaise, ...

Ces demandes visent à rétablir une certaine diversité dans l'encépagement ouvrant droit au bénéfice de l'AOC, tout en maintenant ou améliorant la qualité des produits par l'association (en tant que cépage accessoire) d'un cépage pouvant présenter par exemple, une sensibilité moindre à la coulure (cas du grenache $\mathrm{N}$ ) ou à Botrytis cinerea (cas du gamay $\mathrm{N}$, alors que le gamaret $\mathrm{N}$ semble présenter une moindre sensibilité liée au caractère peu compact des grappes et à l'épaisseur des pellicules, caractère que ce cépage semble combiner avec d'autres qualités - bon niveau de maturité, richesse en composés phénoliques notamment).

Des demandes de cette nature ont bénéficié d'avis favorables, d'autres sont en cours d'étude.

c) Les demandes concernant des cépages issus de croisements interspécifiques entre l'espèce Vitis vinifera et d'autres espèces Vitis.

Des structures techniques et de recherche travaillent actuellement sur l'obtention de nouveaux croisements interspécifiques, dits croisements «d'absorption », entre des vignes de l'espèce Vitis vinifera et des vignes d'autres espèces, lesquelles apportent des gènes de résistance à certaines maladies fongiques comme par exemple le mildiou ou l'oïdium.

L'objectif est d'obtenir, après un certain nombre de rétrocroisements, d'une part le maintien des caractéristiques du cépage appartenant à Vitis vinifera, et d'autre part d'introduire les gènes de résistance aux maladies dans la descendance.

A moyen terme il est fort probable que pourront être sélectionnées « de nouvelles obtentions variétales »durablement résistantes aux principales maladies fongiques. Ces nouvelles obtentions pourraient faire l'objet de demandes de la part des producteurs d'IG.

\section{Les décisions actuelles de I'INAO relatives aux demandes d'évolution de l'encépagement}

\subsection{Les décisions de 2009}

En 2009 l'INAO a conclu à la réaffirmation de tout l'intérêt qu'il convient de porter aux initiatives d'obtention de nouveaux 《cépages », dés lors que ceux-ci vont maintenir et réaffirmer la 《typicité » des AOC, et donc leur lien au terroir.

Cette dernière exigence nécessite d'évaluer ces nouveaux cépages dans le cadre d'expérimentations conduites selon un protocole rigoureux mettant en œuvre les autres conditions de production du cahier des charges de l'AOC, et ce durant une période suffisante, fixée à 10 ans minimum. Cette durée pourra toutefois être adaptée 
en fonction du nombre de sites d'expérimentation situés dans l'aire de l'AOC.

L'évaluation des potentialités d'une nouvelle obtention et des caractéristiques des produits obtenus doit se faire en comparaison avec le ou les cépage(s) principaux.

Les expérimentations doivent être réalisées dans les différentes conditions de milieu naturel pouvant exister au sein de l'aire de production de l'appellation.

Toute introduction d'un nouveau cépage sera limitée à $10 \%$ maximum de l'encépagement de l'AOC concernée, ce qui permet de disposer d'un recul suffisant tout en limitant les incidences sur la typicité finale du produit.

Enfin les produits issus de ce nouveau cépage doivent être utilisés obligatoirement assemblés avec les autres cépages de l'appellation, et sans étiquetage spécifique de ce nouveau cépage.

L'INAO a également rappelé que rien ne s'opposait à admettre de nouveaux croisements intraspécifiques.

Concernant les croisements d'absorption (croisements interspécifiques), le principe d'intégration de ces nouvelles obtentions variétales en AOC a été accepté, et l'INAO a également confirmé que les recherches dans ce domaine devaient être poursuivies, après de nombreux débats.

Au sein même de l'OIV des discussions animées sur la place à donner au regent $\mathrm{N}$ (diana $\mathrm{B} \mathrm{x}$ chambourcin $\mathrm{N}$ ) ont eu lieu.

Finalement l'INAO a confirmé que les recherches concernant les cépages interspécifiques devaient être poursuivies.

Ces recherches et expérimentations sont d'autant plus importantes face aux mesures qui seront à prendre à moyen terme pour tenir compte des restrictions imposées à l'usage des intrants (pesticides, irrigation, ...) sous la pression de la réglementation et de l'opinion publique. Il est indispensable de favoriser les travaux alternatifs à la lutte chimique, seule réponse immédiate aux contraintes environnementales.

Il faudra également favoriser l'obtention des cépages plus résistants à des conditions climatiques plus contraignantes (sécheresse, températures caniculaires, résistance au vent, ...), qui devraient probablement être plus fréquentes à l'avenir.

Il conviendra parallèlement de favoriser une politique de conservation des cépages historiques qui ont pu à l'époque être abandonnés ou délaissés pour différents motifs (productivité jugée trop faible, faible teneur en sucre des raisins, forte sensibilité à telle ou telle maladie maintenant maitrisée, ...) jugés aujourd'hui moins rédhibitoires, plutôt qu'à la faible qualité de leur production.

Une sélection massale bien conduite pourra également présenter un intérêt dans le choix du matériel végétal, et il convient de ne pas négliger ce type de solution.

Enfin en l'état actuel des connaissances, le recours à la transgénèse et aux OGM reste exclu pour les appellations d'origine contrôlées. Cette décision confirmait l'avis émis en avril 2001, qui précisait que la transgenèse devait rester uniquement un outil de recherche, scientifique et exploratoire.

\subsection{Certaines questions restent à préciser}

a) Le principe d'une éventuelle incorporation d'un cépage interspécifique dans une AOC a été acté par les instances de l'INAO, sous certaines conditions.

La réglementation européenne (RCE 1308-2013, article 93) actuellement en vigueur précise que l'encépagement des appellations d'origine (AOP) est réservé exclusivement aux variétés de vigne appartenant à la seule espèce Vitis vinifera, alors qu'un vin bénéficiant d'une IGP peut être élaboré à partir de variétés de vigne de l'espèce Vitis vinifera ou issues d'un croisement entre ladite espèce et d'autres espèces du genre Vitis.

Une éventuelle demande d'introduction dans l'encépagement d'une AOC (AOP) d'un cépage issu d'un croisement interspécifique ne pourra donc être envisagée qu'après une modification de la réglementation européenne.

Une éventuelle modification de la réglementation n'interviendra dans tous les cas qu'après un certain délai, délai qui pourra être mis à profit pour réaliser les différentes études requises pour l'incorporation de nouveaux cépages.

b) Les caractéristiques d'acquisition de ces résistances aux maladies des cépages interspécifiques devront être connues, afin de pouvoir répondre plus précisément à d'éventuelles difficultés relatives à des phénomènes de contournement de résistance.

c) Pour toutes les nouvelles obtentions, intra ou interspécifiques, il conviendra de s'assurer que les dénominations retenues pour ces nouvelles obtentions ne permettront pas un détournement de notoriété, notamment dans le cas d'une incorporation dans le nom de ce nouveau cépage d'un nom géographique faisant référence à telle ou telle indication géographique de forte notoriété.

\section{Les prochains défis auxquels devront faire face les indications géographiques}

Les indications géographiques vont devoir répondre à un certain nombre de questions dans un avenir plus ou moins proches, et notamment :

- l'adaptation du vignoble aux conditions générées par les évolutions climatiques,

- une meilleure adaptation aux contraintes environnementales, avec notamment la probable réduction des intrants pouvant être utilisés (produits phytosanitaires, eau, ... ), et la préservation de la biodiversité,

- une réponse aux demandes des consommateurs portant sur l'évolution de la gamme des produits en adéquation à la demande du consommateur (vin plus léger, plus fruité,....), et la réduction des teneurs en alcool.

\subsection{S'adapter aux évolutions climatiques}

La composante climatique étant une des composantes essentielles du terroir, les prochaines évolutions climatiques auront des conséquences sur les caractéristiques des vins 
produits et tout particulièrement ceux présentant un lien fort avec leur terroir.

Toutes les études consultées s'accordent sur une augmentation générale des températures, qui pourra toutefois être plus ou moins importante selon les régions. Cette hausse des températures s'accompagnera (sauf en cas de stress hydrique trop marqué) d'une avancée de la phénologie de la vigne, et donc d'un avancement plus ou moins significatif des dates de vendanges selon les situations.

Les prévisions relatives aux évolutions des précipitations sont à l'heure actuelle beaucoup moins précises, même si l'on peut retenir très schématiquement une baisse des précipitations dans les régions méridionales et leur accentuation dans les zones plus septentrionales.

Les études laissent présager également une grande variabilité annuelle des caractéristiques climatiques, ainsi qu'un fort développement des épisodes climatiques extrêmes (sécheresse prolongée, orages violents, ... ).

Les évolutions climatiques seront donc plurielles, avec des amplitudes plus ou moins fortes selon les régions, et des conséquences pouvant être soit favorables (avec l'obtention de maturités plus accomplies), soit défavorables.

Elles nécessiteront des réponses variables selon les indications géographiques.

D’une manière générale, on peut considérer en première approche que ces évolutions entraineront une modification des caractéristiques des vins produits, avec des hausses de la teneur en alcool des vins, des modifications des équilibres acidité/alcool, de la structure tannique ou encore des composantes aromatiques des vins,

Afin de limiter les conséquences des évolutions climatiques et maintenir les caractéristiques des vins, des stratégies d'adaptation pourront être mises en place dans les différentes indications géographiques, et ce dans des pas de temps plus ou moins rapides.

Les premières adaptations à pouvoir être mises en œuvre porteront sur les pratiques culturales, avec notamment une réflexion sur le rapport surface foliaire/poids de récolte, qui pourrait être abaissé par rapport à la situation actuelle, avec pour objectif final une teneur en sucre des raisins moins élevée.

Ces adaptations pourront d'autant plus facilement être mises en œuvre que depuis 30 ans l'INAO a conduit une politique portant sur l'amélioration de la maturité des raisins, se traduisant par une évolution des pratiques culturales inscrites dans les cahiers des charges des AOC (limitation du nombre de bourgeons à la taille, réduction des rendements autorisés, définition de surfaces foliaires minimales, ... ).

L'obtention d'une meilleure maturité des raisins a également été favorisée par la modification d'autres critères : utilisation de porte-greffes moins productifs, baisse importante de la fertilisation, réalisation d'opérations d'effeuillage, vendanges en vert, enherbement de certaines vignes, ...

Les conséquences des évolutions climatiques sur les caractéristiques des vins produits pourraient ainsi être facilement limitées en adaptant certaines pratiques actuelles.

Le cas échéant une adaptation par l'INAO du contenu des cahiers des charges pourra être réalisée.

A plus long terme, une autre réponse pourra être apportée avec la recherche et l'expérimentation de cépages plus tardifs, permettant de retarder les périodes de vendanges et de limiter ainsi les inconvénients dus à des vendanges effectuées dans des périodes trop chaudes en plein été.

Nous avons vu précédemment que les procédures pour expérimenter de nouveaux cépages étaient déjà en place pour les AOC.

Les expérimentations devront toutefois démontrer le maintien de l'originalité des produits d'AOC, pour permettre le cas échéant l'intégration de nouveaux cépages dans les cahiers des charges.

\subsection{Favoriser la réduction des intrants utilisés dans la culture de la vigne}

Une autre interrogation porte sur la probable nécessité de réduire l'utilisation des intrants dans la culture de la vigne (produits phytosanitaires, fertilisants ou encore irrigation).

La limitation de l'utilisation d'intrants devrait s'intensifier dans les prochaines années avec de probables évolutions de la réglementation, en relation avec les préoccupations environnementales croissantes de la société.

Une des réponses sera alors de pouvoir disposer de cépages présentant une résistance plus grande aux différentes maladies de la vigne.

Les conséquences de cette probable réduction des intrants seront également à rapprocher des évolutions possibles des caractéristiques des maladies de la vigne (maladies cryptogamiques, insectes, acariens, ...), en relation avec les évolutions climatiques.

Les différents travaux de recherches menées actuellement sur l'obtention de nouveaux cépages, plus résistants aux principales maladies, mieux adaptés à des conditions climatiques extrêmes ou permettant de mieux préserver la biodiversité, ouvrent de nouvelles pistes de réflexion aux producteurs des différentes IG.

Comme nous l'avons vu précédemment les décisions prises par l'INAO offrent la possibilité de tester de nouveaux cépages en IG, AOP ou IGP, la responsabilité des demandes restant au final du ressort des producteurs.

\subsection{Prendre les évolutions des modes de consommation du vin}

Nous observons aujourd'hui un développement rapide de marchés pour de nouveaux produits, avec une demande orientée sur des vins plus légers, fruités, ..., sur des couleurs différentes.

L'apparition de ce type de demandes, adaptées à des modes de consommation en perpétuelle évolution, a incontestablement modifié la physionomie des vignobles dans un certain nombre d'indications géographiques, et plus particulièrement dans les IGP.

L'utilisation de méthodes correctives basées sur des procédés physiques permettant une réduction des teneurs en sucres des moûts ou des teneurs en alcool des vins, est 
une première possibilité de réponse à ces enjeux de santé publique.

Mais l'utilisation de cépages permettant de prévenir 《 naturellement » un potentiel élevé d'alcool est également une réponse adaptée à l'émergence de ces nouveaux marchés.

\subsection{Intégrer le rôle des politiques de santé publique}

Si des effets bénéfiques de consommation modérée de vins doivent faire l'objet de travaux de recherche scientifique pour les consolider, si cette thématique doit faire l'objet d'une communication appropriée, certaines politiques de santé publique conduiront au moins à privilégier les cépages participant à la réduction des teneurs en alcool, ou à se prêter facilement à des techniques de désalcoolisation.

Ces politiques sont d'intensité variable selon les pays, mais les tendances générales peuvent conduire à s'interroger, au moins face à certains marchés, sur la stratégie à retenir.

\section{Conclusion}

Les indications géographiques viticoles françaises ont régulièrement eu l'occasion de démontrer leur capacité à répondre à de nouvelles problématiques.

$\mathrm{Si}$ les IGP possèdent par définition un espace de liberté leur permettant d'évoluer dans un pas de temps rapide, les AOC ont également démontré une aptitude à gérer et intégrer un certain nombre d'innovations, sous réserve que celles-ci permettent de continuer à produire des vins respectant l'originalité qui avait contribué à leur reconnaissance.

Les innovations variétales feront partie de la panoplie mise à disposition des producteurs pour répondre aux principales problématiques qui se poseront dans un proche avenir, et notamment celles portant sur l'adaptation aux évolutions climatiques, la réduction des intrants utilisés ou encore l'apparition de nouveaux marchés portant sur des vins à teneur réduite en alcool.

\section{Références}

Joseph CAPUS : La genèse des appellations contrôlées Actes du 1er congrès national de l'origine (Deauville, 2527 juin 1948)

Actes du $7^{\text {ème }}$ congrès national de l'origine (Bordeaux, 12 au 15 mai 1950)

Germain LAFFORGUE : Le vignoble girondin, préface et introduction de Joseph CAPUS (1947, Editions Louis LARMAT)

Jean-Pierre DOAZAN (INRA Bordeaux) : Réflexion sur l'évolution de l'encépagement pour la production de vin de qualité, en France

Jean-Philippe ROBY - Louis BORDENAVE : Evolution du matériel végétal viticole - Constat et perspectives d'avenir (Terroirs viticoles - 11 décembre 2008)

Présentations effectuées au séminaire LACCAVE : La vigne et le vin face aux changements climatiques Impacts et adaptation pour le vignoble français (Montpellier 21 mars 2014)

Dossiers étudiés au Comité national des Vins et Eau de Vie de l'INAO:

Octobre 1989 : Utilisation de variétés de vignes issues de croisements interspécifiques ou intraspécifiques (dossier 6745)

Novembre 2002 : Rapport de la commission d'enquête nationale : Introduction de nouveaux cépages en appellations d'origine viticoles (dossier 02 -515)

Septembre 2009 : Classification du matériel végétal de l'espèce Vitis vinifera pour les AOC - Possibilité d'introduction en AOC de créations variétales issues de nouveaux croisements (dossier 2009 - 306) 